\title{
X-ray reverberation lags from the 1.5 Seyfert galaxy NGC 5273
}

\author{
F. M. Vincentelli ${ }^{\oplus},{ }^{1 \star}$ G. Mastroserio, ${ }^{2}$ I. McHardy, ${ }^{1}$ A. Ingram ${ }^{\oplus 3}$ and M. Pahari ${ }^{\oplus 1}$ \\ ${ }^{1}$ Department of Physics and Astronomy, University of Southampton, Highfield, Southampton SO17 1BJ, UK \\ ${ }^{2}$ Astronomical Institute, Anton Pannekoek, Univerity of Amsterdam, Science Park 904, NL-1098 XH Amsterdam, The Netherlands \\ ${ }^{3}$ Department of Physics, Astrophysics, University of Oxford, Denys Wilkinson Building, Keble Road, Oxford OX1 3RH, UK
}

Accepted 2019 December 10. Received 2019 December 10; in original form 2019 September 16

\begin{abstract}
We present the results of X-ray spectral-timing analysis of a $90 \mathrm{ks} X M M-N e w t o n$ observation of the nearby, broad-line, early-type AGN NGC5273. The X-ray spectrum revealed the clear presence of a reflection component at high energies, with a clear signature of a narrow iron line at $6.4 \mathrm{keV}$, consistent with distant reflection. Applying a relativistic reflection model, we found only marginal evidence for a broader relativistic line component. However, crossspectral analysis revealed that, between 4 and $6 \times 10^{-4} \mathrm{~Hz}$, the $5-8 \mathrm{keV}$ band lagged the 2-3 keV band, implying reflection of the iron line from material close to the black hole. From the analysis of the lag-energy spectrum, we found a broad, but skewed line with a peak of $\approx 1000 \mathrm{~s}$ at $7.5 \mathrm{keV}$ relative to the continuum, which we interpret as the iron line in the reverberation spectrum from an illuminated accretion disc. From the asymmetry in the shape of lag-energy spectrum, we also found that the source is consistent with having an inclination $\geq 45^{\circ}$.
\end{abstract}

Key words: accretion, accretion discs.

\section{INTRODUCTION}

Active galactic nuclei (AGNs) are believed to originate from accretion of matter on to supermassive black holes $\left(10^{6}-10^{9} \mathrm{M}_{\odot}\right)$ at the centres of galaxies. They produce emission from radio up to gamma-rays, with a complex spectrum deriving from a number of different physical components (Fabian 2012; Padovani et al. 2017). Strong outflows, and the copious amounts of radiation produced by these objects are observed to affect the properties of their host galaxies (Schawinski et al. 2007; Cicone et al. 2014; King \& Pounds 2015). Most of our understanding, however, is still limited by large uncertainties concerning the precise geometry of these systems. Constraining physical parameters such as black hole mass and disc inclination is therefore crucial to understand the underlying physical processes and their consequences on larger scales (see e.g. Bentz et al. 2014; Graham et al. 2011).

Historically, the study of the X-ray emission and its variability have proven to be among the best approaches for shedding light on the physical processes taking place in the innermost regions, close to central compact object. The primary $\mathrm{X}$-ray radiation is thought to be produced in a hot electron corona by Compton upscattering lower energy disc photons (Haardt \& Maraschi 1991). This radiation illuminates the surrounding relatively cold accretion disc (Guilbert \& Rees 1988; Fabian et al. 1989), giving rise to a characteristic reflection spectrum (Ross \& Fabian 1993; García

\footnotetext{
^E-mail: F.M.Vincentelli@ soton.ac.uk
}

et al. 2013). One of the most prominent features of this reflection spectrum is the presence of the iron $\mathrm{K} \alpha$ line at $6.4 \mathrm{keV}$. In type- 1 Seyfert galaxies, this feature was observed to be broadened and skewed, which was successfully explained in terms of general relativistic effects in the strong gravitational field near to the central black hole (Tanaka et al. 1995).

$\mathrm{X}$-ray variability is known to be a fundamental property of AGNs which has enabled the extraction of key information on the physical properties of these systems (Uttley \& McHardy 2001; Uttley, McHardy \& Vaughan 2005; Vaughan et al. 2003). The timescales on which the high-energy emission of these sources can vary span from years down to $\approx 100$ s (Lawrence et al. 1987; McHardy \& Czerny 1987). The Fourier power spectral density measured from the emission of these sources is characterized by a decreasing broken power-law trend (e.g. the slope increases from $\approx-1$ at lower frequencies to $\approx-2$ at higher frequencies) with a break frequency which scales with the mass (Uttley, McHardy \& Papadakis 2002; McHardy et al. 2004, 2006).

Recent long and continuous exposures performed by $X M M$ Newton have permitted detailed study of the X-ray cross-spectral properties of AGN, down to $\approx 0.5 \mathrm{keV}$, on very short time-scales. The main result from this approach has been the discovery of a lag between the X-ray continuum and the radiation emitted both in the iron emission lines and in the low-energy $(<2 \mathrm{keV})$ reflection continuum. In particular Fabian et al. (2009) produced a firm detection in 1H 0707-495 that the variability in the iron L line (0.3$1 \mathrm{keV}$ ) lagged the rest of the continuum. Marginal evidence for a soft lag in the same energy range had already been found by Markowitz 
et al. (2007) and McHardy et al. (2007) for Mkn 766 and Ark 564, respectively. Further observations revealed the presence of such a lag in other sources both in the low-energy continuum and also in the $\mathrm{K} \alpha$ line (Emmanoulopoulos, McHardy \& Papadakis 2011; Zoghbi, Uttley \& Fabian 2011; Zoghbi et al. 2012, 2013; Kara et al. 2013a,b). A correlation between the mass and the amplitude of the lag among several Seyfert type-1 galaxies (De Marco et al. 2013; Kara et al. 2016) was discovered, suggesting a common geometry among these sources. These results motivated more quantitative modelling of the reverberation phenomenon. Initially, the work focused on reproducing the Fourier-resolved lags between two X-ray energy bands using analytic response functions for different geometries (Emmanoulopoulos, McHardy \& Papadakis 2011; Alston, Done \& Vaughan 2014). More detailed work (e.g. Emmanoulopoulos et al. 2014; Cackett et al. 2014) included response functions based on numerical General Relativistic ray tracing to determine black hole mass, spin, inclination, and X-ray source height assuming the lamppost model. More recently, it has become possible to also include the energy dependence of the lags in the models. This has allowed the properties of the accretion process to be investigated more comprehensively, including additional parameters such as the shape of the X-ray source (lamp-post or extended corona, Cackett et al. 2014; Wilkins et al. 2016; Chainakun \& Young 2017; CaballeroGarcía et al. 2018; Taylor \& Reynolds 2018; Chainakun et al. 2019; Ingram et al. 2019).

NGC 5273 is a nearby $(z=0.00362$, Bentz et al. 2014) lowluminosity AGN (LLAGN) hosted by a lenticular galaxy (S0 morphology) at a distance of $16 . \pm 1.6 \mathrm{Mpc}$ (Tonry et al. 2001). First known as a 1.9 Seyfert Galaxy (Osterbrock \& Martel 1993), it has recently been reclassified as 1.5 Seyfert galaxy after a re-analysis of the contribution of the host galaxy to its spectrum revealed the presence of broad components to $H_{\alpha}$ and $H_{\beta}$ (Trippe et al. 2010). Through optical reverberation mapping measurements, Bentz et al. (2014) measured a black hole mass of $4.7 \pm 1.1 \times 10^{6} \mathrm{M}_{\odot}$.

X-ray observations with Suzaku showed that NGC 5273 was significantly variable in X-rays (Kawamuro et al. 2016). Recent analysis of a Swift+NuSTAR observation by Pahari et al. (2017) found clear evidence for non-relativistic reflection in the X-ray spectrum, with also a marginal detection of a high-energy cut-off at $140 \mathrm{keV}$. In this work, we analyse a $90 \mathrm{ks} X M M-$ Newton X-ray observation, confirming rapid short time-scale variability (Section 2). In Section 3 , we analyse the time-averaged $X$-ray spectrum under various model assumptions, looking also at the X-ray interband lags. We then discuss the implications of these results for the source geometry in Section 4.

\section{DATA REDUCTION}

Data analysed in this work were taken in Full Frame mode with the EPIC-pn camera on board of the XMM-Newton satellite during a 90 ks pointing performed on 2017 June 02 (OBSID 0805080401). Events were extracted within a circle of 45 arcsec radius around the source and filtering events with PATTERN $<=4$ and FLAG $==0$ . Events were then binned in a light curve with a time resolution of $100 \mathrm{~s}$ (see Fig. 1). The source was found to vary significantly within the observation, with a mean count rate in the energy range $0.5-10 \mathrm{keV}$ of $0.361 \pm 0.003$ counts s${ }^{-1}$ and a fractional rms of 0.16 . In order to quantify the background contribution, events were extracted from a clear circular region in the field with the same aperture size as the source region. Two small segments (each of duration $3 \mathrm{ks}$ ) in which the background count rate exceeded 0.04 counts $\mathrm{s}^{-1}$ were excluded from the analysis. The same two source

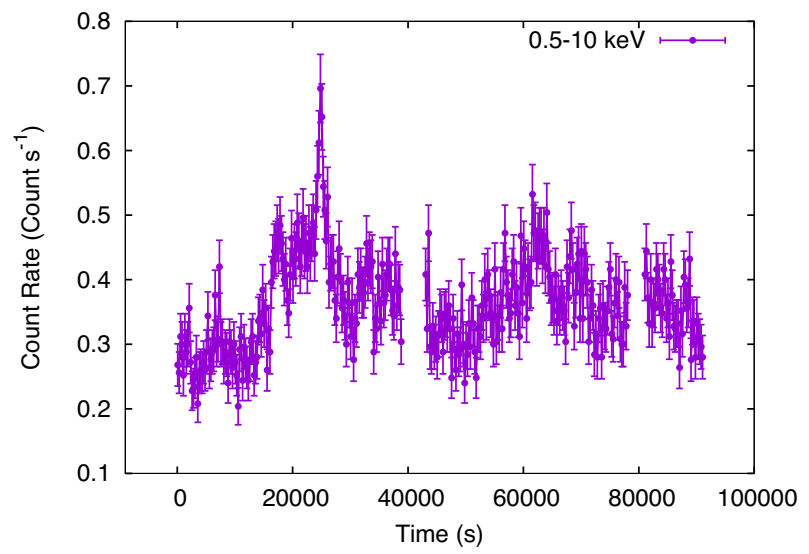

Figure 1. X-ray $(0.5-10 \mathrm{keV})$ light curve extracted using the EPIC-pn data Time resolution is of $100 \mathrm{~s}$.

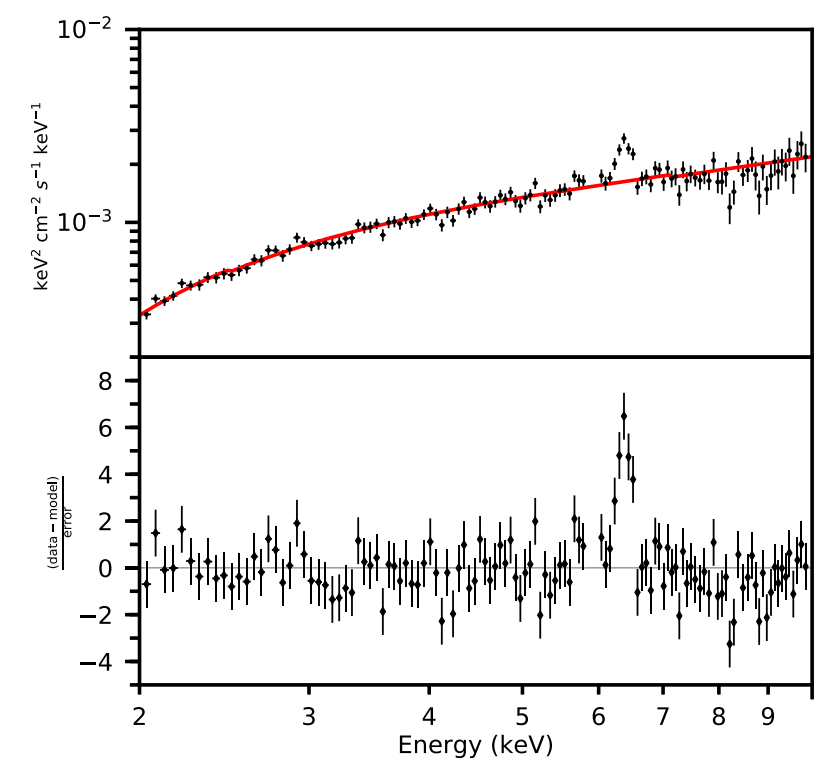

Figure 2. Unfolded spectrum (upper panel) and the residuals (bottom panel) of the best fit using only an absorbed power law in the energy range 2-10 keV. An excess at $6.5 \mathrm{keV}$ is evident.

and background regions were used to extract the time-averaged energy spectrum. The energy spectrum was then grouped in order to have a minimum of 20 counts per energy bin. On top of the statistical errors, we added 1 per cent systematic errors when fitting the time-averaged energy spectrum.

\section{DATA ANALYSIS}

\subsection{Spectral analysis}

The time-averaged energy spectrum is strongly absorbed at energies below $2 \mathrm{keV}$. We therefore first considered the time-averaged spectrum between 2 and $10 \mathrm{keV}$. Fig. 2 shows the residuals of fitting an absorbed power-law (TBABS+POWERLAW), with the Galactic hydrogen column density fixed to $n_{\mathrm{H}}=9.2 \times 10^{19} \mathrm{~cm}^{-2}$ (Pahari et al. 2017). All the spectral models reported throughout this paper use this same fixed hydrogen column density to account for absorption in our galaxy. The residuals show a significant narrow excess between 6 and $7 \mathrm{keV}$ (Fig. 2), suggesting the presence of a 


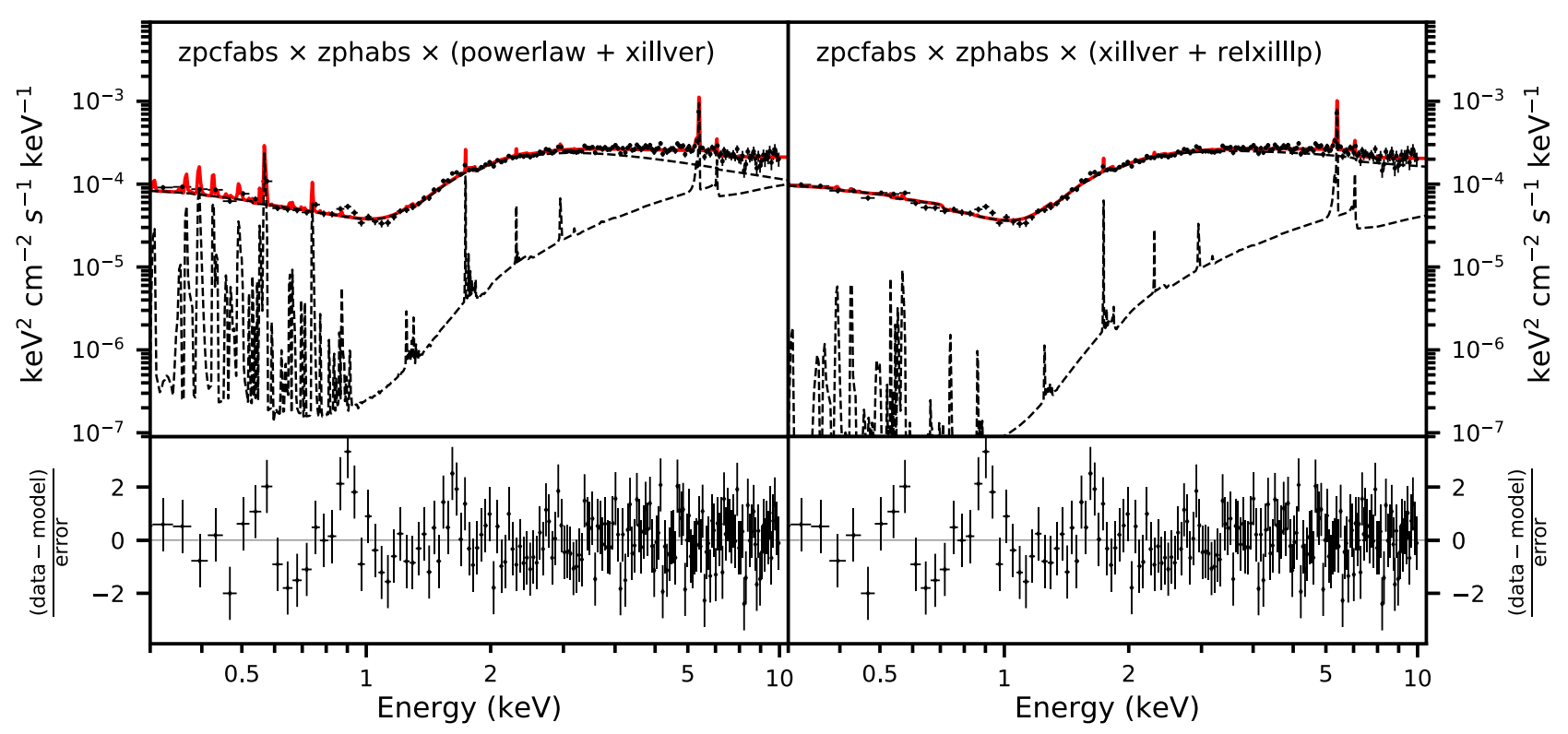

Figure 3. Unfolded spectra with the best-fitting models (red line) and its residuals using an absorbed power-law and XILLVER (left-hand panels) and using RELXILLLP + XILLVER (right-hand panels) in the energy range $0.3-10 \mathrm{keV}$. The components of each models are listed in the plots and represented with dashed lines.

non-relativistic, distant reflection component (see also Pahari et al. 2017). We modelled this feature using the XILLVER model (García et al. 2013) and using the power-law model for the inverse-Compton emission. We then also included the softer $0.3-2 \mathrm{keV}$ energy range. The soft part of the time-averaged spectrum shows strong absorption due to gas of the host galaxy in our the line of sight. We modelled this extra absorption component with the partial covering model ZPCABS which accounts for the cosmological redshift of the galaxy $(z=0.00362)$. Following the procedure described in Pahari et al. (2017), we fixed the inclination $\left(i=40^{\circ}\right)$ because the spectral fit is not very sensitive to this parameter. The illuminating power-law index of XILLVER is fixed to the power-law index representing the direct emission, and the same procedure is applied to the highenergy cut-off, which is fixed to $140 \mathrm{keV}$ (Pahari et al. 2017) because the energy range of XMM-Newton is too low to put a reliable constraint on this parameter. Top panel in Fig. 3 shows the unfolded spectrum with the best-fitting model (red line), with the different components (dotted lines). The bottom panels show the residuals of the fit. Addition of the reflection component improves the residuals structure in the iron line energy range and leads to an acceptable reduced $\chi^{2}$ of $180.5 / 163$.

We measured a total flux between 0.5 and $10 \mathrm{keV}$ of $3.3_{-0.1}^{+0.01} \times$ $10^{-12} \mathrm{erg} \mathrm{cm}^{-2} \mathrm{~s}^{-1}$; in the $0.5-2$ and $2-10 \mathrm{keV}$ bands we measured respectively $1.9_{-0.06}^{+0.02} \times 10^{-13}$ and $3.1_{-0.1}^{+0.03} \times 10^{-12} \mathrm{erg} \mathrm{cm}^{-2} \mathrm{~s}^{-1}$. The second column of Table 1 reports the parameter values of the model. In order to model the strong soft absorption, the partial covering model (ZPCF) has a very high covering factor $\left(f_{\mathrm{c}}=0.958_{-0.006}^{+0.003}\right)$ with a hydrogen column density of $N_{\mathrm{H}}=2.51_{-0.05}^{+0.05} \times 10^{22} \mathrm{~cm}^{-2}$. We note that the residuals present some excesses for energies lower than $2 \mathrm{keV}$, in particular apparent absorption features seem to be present at $\approx 0.7$ and $\approx 1.3 \mathrm{keV}$. Similar features were already seen in the highly absorbed type 2 Seyfert Galaxy IRAS 18325-5926 (Lobban, Alston \& Vaughan 2014), but may also be due to variable abundances in addition to iron. A more detailed modelling, however, is beyond the scope of this paper. The index of the illuminating power law is $\Gamma=1.86_{-0.01}^{+0.01}$ which is very similar to the value Pahari et al. (2017) found with Swift/XRT and NuSTAR $\left(\Gamma=1.81_{-0.03}^{+0.02}\right)$.
Table 1. Best-fitting parameter values for time-averaged energy spectrum. The models considered in the fit are specified in the table. The hydrogen column density for the galactic absorption, the high-energy cut-off and the spin of the black hole are fixed to $0.0092 \times 10^{22} \mathrm{~cm}^{-2}, 140 \mathrm{keV}$, and 0.998 respectively. The errors are reported at 90 per cent confidence level.

\begin{tabular}{lcc}
\hline Parameter/model & $\begin{array}{c}\text { ZPCF } \times \text { TBABS } \times \\
(\mathrm{PW}+\mathrm{XILLVER})\end{array}$ & $\begin{array}{c}\mathrm{ZPCF} \times \mathrm{TBABS} \times \\
(\mathrm{REL}+\mathrm{XIL})\end{array}$ \\
\hline$n_{\mathrm{H}}\left[10^{22} \mathrm{~cm}^{-2}\right]$ (parCov) & $2.52_{-0.05}^{+0.05} \times 10^{22}$ & $2.41_{-0.05}^{+0.05} \times 10^{22}$ \\
$f_{\mathrm{c}}(\mathrm{parCov})$ & $0.958_{-0.004}^{+0.003}$ & $0.961_{-0.005}^{+0.005}$ \\
$h\left[R_{\mathrm{g}}\right]$ & - & $3.05_{-0.09}^{+0.09}$ \\
$r_{\text {in }}[I S C O]$ & - & $1.02_{-c}^{+0.17}$ \\
$\Gamma_{\mathrm{pl}}$ & $1.88_{-0.01}^{+0.01}$ & $1.77_{-0.01}^{+0.01}$ \\
$\log \xi^{(a)}$ & $0.31_{-0.10}^{+0.03}(\mathrm{XIL})$ & $2.69_{-0.22}^{+0.35}(\mathrm{REL})$ \\
$A_{\mathrm{Fe}}$ & $0.5_{b}^{+0.1}$ & $0.9_{-0.1}^{+0.3}$ \\
refl_frac & - & $0.99_{-0.14}^{+0.16}$ \\
red $\chi^{2}\left(\chi^{2} /\right.$ dof $)$ & $180 / 163$ & $175 / 160$ \\
\hline
\end{tabular}

Notes: ${ }^{a}$ In the XILLVER + RELXILL model XILLVER's ionization is 0.0 .

${ }^{b}$ The lower limit of the iron abundance is 0.5 .

${ }^{c}$ The lower limit is at ISCO.

The iron abundance of the reflection model is pegged to the lowest allowed value of XILLVER (0.5), whereas it is close to unity in the model of Pahari et al. (2017) (1.2 \pm 0.3$)$. The distant reflecting plasma seems to have a very low ionization $\left(\log \xi=0.33_{-0.03}^{+0.04}\right.$ ), as expected far from the black hole. As a further test we also reperformed the fit leaving free the high-energy cut-off. However, no significant variation was found.

Many AGNs show an additional broad relativistic reflection component around the iron $K \alpha$ energy in their time-averaged spectra. We therefore also used the RELXILLP model (Dauser et al. 2014; García et al. 2014) which accounts for both the direct and the reflected emission. The former is described with a cut-off power-law and the latter is the radiation emitted from a lamp-post source above the black hole and reflected by a geometrically thin 
but optically thick accretion disc. We consider a constant radial ionization profile in the accretion disc. The parameters shared by the distant reflection and the relativistic reflection emission such as the power-law index of the illuminating radiation, its highenergy cut-off, the iron abundance in the disc and the inclination of the system are tied together. Except for the high-energy cut-off and the black hole spin $(a)$, these parameters are free in the fit, as are the height of the source $(h)$, the inner radius of the disc and the reflection fraction (refl_frac). Even though black hole spin is known to affect the reflection spectrum (see e.g. Martocchia, Karas \& Matt 2000), the model is more sensitive to the inner disc radius than the actual spin value (Dauser et al. 2013). Here, we are more interested in the extension of the accretion disc, therefore we consider a maximally spinning black hole with a free inner radius parameter. Thus we allow for the possibility that the accretion disc does not reach in as far as the last stable circular orbit (ISCO). The distant reflector is considered neutral so its ionization parameter is set to 0 . Table 1 shows the values of the parameters for the best-fitting model. We note that using two refection components (non-relativistic and relativistic reflection) improves the reduced $\chi^{2}$. However, both models are statistically acceptable and the $F$ test shows that the relativistic component is significant with $2 \sigma$ confidence.

\subsection{Long-term spectral evolution}

The flux obtained with spectral analysis was found to be roughly a factor $\approx 5$ times higher than the one measured by Pahari et al. (2017) $3 \mathrm{yr}$ before in 2014 July. On the other hand, the estimated spectral parameters from the two data sets were found be in good agreement, and the ratio of the $0.5-2$ and the $2-10 \mathrm{keV}$ fluxes does not show significant variations between the two epochs. With this in mind, we also looked for other archival observations in the $0.5-10 \mathrm{keV}$ band. We found that the source was observed once previously by XMMNewton (Cappi et al. 2006) and by Suzaku (Kawamuro et al. 2016) in 2002 and 2013, respectively. The reported flux values indicate that only the first observation, performed more that $10 \mathrm{yr}$ before the other three, presents a significant difference in the $0.5-2 / 2-10 \mathrm{keV}$ flux ratio compared to the previous results $(\approx 0.2$ versus $\approx 0.05)$. This is mainly due to a significantly lower absorption seen in the 2002 epoch. Past observations with the ROSAT satellite in the soft band also showed significant variations (Polletta et al. 1996), consistent with those observed in the recent years. The long-term light curve in two bands is plotted in Fig. 4 and shows how the X-ray emission changes significantly over the $\approx 30 \mathrm{yr}$ of observations, and in this last observation the source was at one of its minimum levels. AGNs are known to be variable on long time-scales. Such variations can be due to obscuring events, which change the spectrum, especially at low Xray energies, or intrinsic variations of the power-law normalization. Given that the latter cause does not affect the slope of the spectrum (and therefore the spectral hardness), this suggests that at least the variations observed in the last $3 \mathrm{yr}$ are intrinsic and and originating in the immediate environment of the primary X-ray source.

\subsection{Timing analysis}

In order to study the variability properties of this data set, we followed the procedures described in Uttley et al. (2014). To do this, we used the open software STINGRAY (Huppenkothen et al. 2019). In particular, we computed the Fourier frequency dependent time lags with respect to the continuum-dominated reference band of 2-3 keV for the two subject bands: $4-5 \mathrm{keV}$ (Fig. 5, blue open

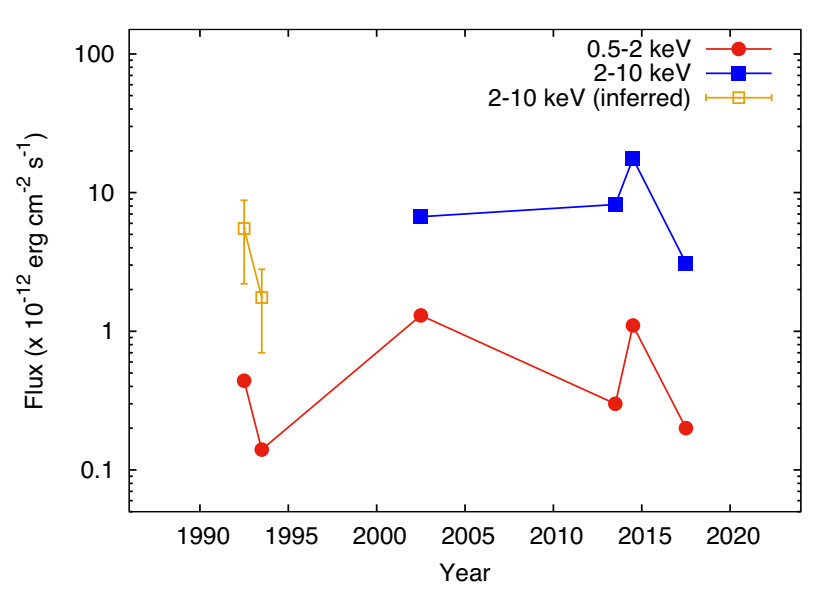

Figure 4. X-ray flux as a function of time collecting archival observations in the $0.5-2$ and 2-10 keV band. The yellow squares are the inferred 2-10 keV flux considering the ROSAT fluxes and the two hardness ratios measured with XMM in 2000 and 2017.

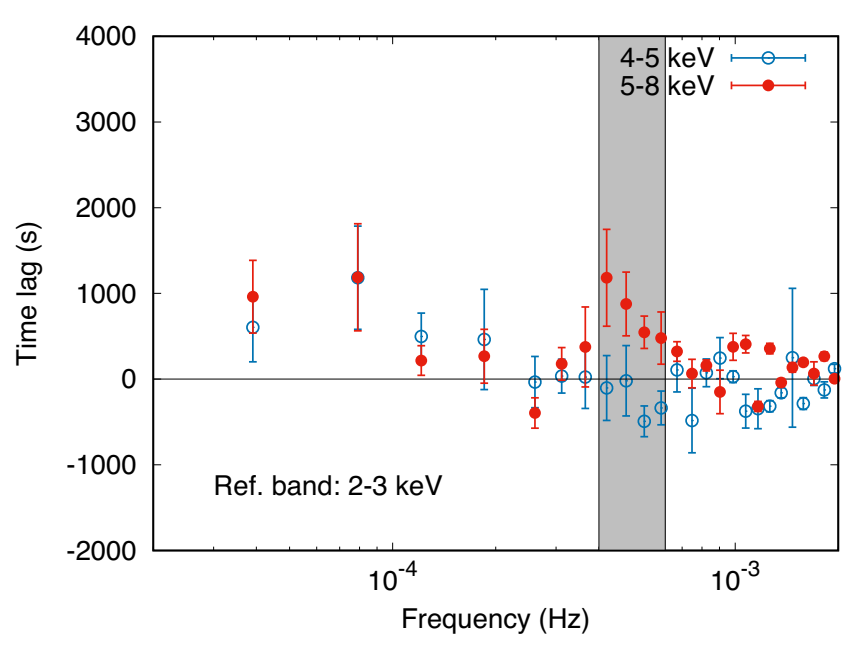

Figure 5. Time lags computed between the 2-3 keV band and the 4-5 (blue open circles) and 5-8 (red filled circles) keV band. Only the latter shows a lag between 4 and $6 \times 10^{-4} \mathrm{~Hz}$ (highlighted in grey).

circles) and 5-8 keV (Fig. 5, red filled circles). In both cases, for lag-frequency spectra for frequencies lower than $10^{-5} \mathrm{~Hz}$, we find a hint of a positive hard lag, possibly due to propagating accretion rate fluctuations. At higher frequencies instead we see a positive lag only between the $2-3$ and the $5-8 \mathrm{keV}$ band, as expected for disc reverberation.

Following all previous papers on lag measurement, we have not corrected the data for possible red noise leakage distortion (see e.g. Jenkins \& Watts 1969) which, if there is significant variability power on time-scales longer than those observed here, might affect the lags. However there is no large-scale trend obvious over the duration of the observed light curve $(\approx 100 \mathrm{ks})$ so red noise leakage may not be large. Moreover we notice that based on the optically determined black hole mass, we do not expect large amplitude variability on time-scales longer than those observed here (McHardy et al. 2006).

From our analysis of the time-averaged spectrum, we select the 2-3 keV band as our reference direct continuum band for the calculation of lags. Here a positive lag means that the harder subject band (i.e. $4-5$ or $5-8 \mathrm{keV}$ ) lags the softer reference band $(2-3 \mathrm{keV})$. In the [4-6] $\times 10^{-4} \mathrm{~Hz}$ frequency range, the $4-5 \mathrm{keV}$ band, which 


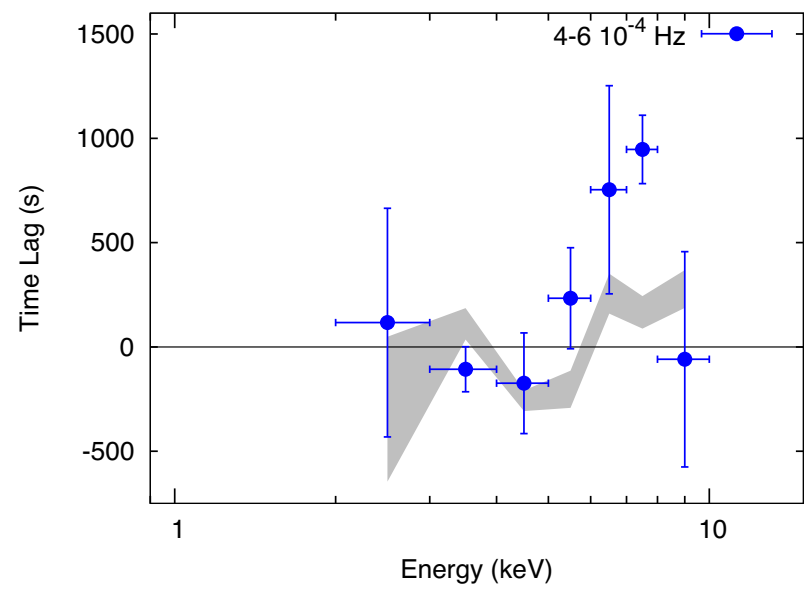

Figure 6. Time lag as a function of the energy. Lags were computed between $4-6 \times 10^{-4} \mathrm{~Hz}$. An excess around $7 \mathrm{keV}$ is clear. Grey area are the lag versus energy computed between $0.9-1.5 \times 10^{-3} \mathrm{~Hz}$.

is dominated by the continuum shows a slightly negative lag, but is consistent with no lag (Fig. 5). A clear positive lag is, however, visible in the same frequency range for the $5-8 \mathrm{keV}$ band that contains the iron line.

For the same [4-6] $\times 10^{-4} \mathrm{~Hz}$ frequency range, we measure the energy dependence of the lags (Fig. 6). To increase the $\mathrm{S} / \mathrm{N}$, we chose as reference band the larger $2-10 \mathrm{keV}$ band, excluding the band relative to which the lag was computed. The lags are consistent with 0 apart from a feature at $\sim 7 \mathrm{keV}$, further indicating the presence of a reverberation lag. The lag versus energy dependence was also computed at higher frequencies $\left(0.9-1.5 \times 10^{-3} \mathrm{~Hz}\right.$, where only marginal evidence of a lag was seen). The grey curve in Fig. 6, shows that for this higher frequency range there is no significant trend which could suggest the presence of reverberation.

In order to quantify our result we also fitted the lag energy spectrum, with a Gaussian line. The best-fitted model has a line centre at $7.02_{-0.22}^{+0.6} \mathrm{keV}$ with $\sigma=0.7_{-0.6}^{+0.3} \mathrm{keV}$.

\section{DISCUSSION}

We have analysed the X-ray spectral-timing properties of the LLAGN NGC 5273 with a 90 ks continuous pointing. We detected for the first time in this source a signature of X-ray reverberation from an accretion disc in the time lags (Figs 5 and 6). On the other hand, we find only marginally significant evidence for a broad iron line in the time-averaged spectrum, such as is expected to arise when the inner edge of the accretion disc is very close to the black hole (Ross \& Fabian 1993; García et al. 2013).

In order to shed light on this inconsistency, we compared the observed lag with that measured in other AGN (De Marco et al. 2013; Kara et al. 2016). For consistency of band selection, we therefore computed the lag between the 3-4 and 5-7 keV bands over the frequency range 4-6 $\times 10^{-4} \mathrm{~Hz}$, finding a lag of $705 \pm 198 \mathrm{~s}$. Assuming a mass for the black hole of $4.7 \times 10^{6} \mathrm{M}_{\odot}$ (Bentz et al. 2014), our measurement is compatible with the mass scaling relationships from (De Marco et al. 2013; Kara et al. 2016) for both lag amplitude and lag frequency. Even though such correlations seem to indicate a common geometry between Seyfert galaxies, they contain substantial scatter, showing that other effects are in play (Kara et al. 2016).
A well known issue in time-lag measurements is 'dilution'. Both the direct and reflected components will be present in both the reference band and the channel of interest. Therefore the measured lag will differ from the intrinsic lag depending on the relative contribution between these two components.

A light crossing time of $700 \mathrm{~s}$ corresponds to a distance of $\approx 20$ gravitational radii $\left(R_{\mathrm{G}}\right)$ for a black hole mass of $\approx 5 \times 10^{6} \mathrm{M}_{\odot}$. Even though it has been shown the X-ray lag cannot be simply converted into light travel distance this value suggests that the disc is truncated. It is perhaps reasonable to expect this source to have a truncated disc, since it has an accretion rate of $\sim 1$ per cent of the Eddington limit (Pahari et al. 2017), which is lower than the accretion rates of most other well-known X-ray bright AGN in which broad iron lines have been found (Kara et al. 2016). The location of the inner edge of the accretion disc as a function of accretion rate is not well measured, but there is evidence that the edge moves outwards as the accretion rate decreases (Cabanac et al. 2009).

Although the detection of a lag in the timing analysis is clear, the detection, or lack of detection, of a broad iron line in the timeaveraged energy spectrum is less clear. The simple fit of ZPCF $\times$ TBABS $\times($ PW + XILLVER $)$, which provides a power law and a narrow iron line, is an acceptable fit ( $\chi^{2}$ of 180/163). As there are no clear residuals showing a broad line above the continuum, if we include a relativistic component (adding RELXILLP), the model prefers a solution with a very broad line and so it concludes that the inner edge of the disc must be at the ISCO. This fit is not significantly better $\left(\chi^{2}\right.$ of 175/160) than the one without a relativistic component (see Section 3.1). Given the relative faintness of NGC 5273 then from the time-averaged spectrum we cannot conclude a great deal about any broad iron line except to say that it is not strong. A truncated disc may therefore provide an explanation of the the lack of a strong iron line in the energy spectrum, even though $F$-test reveals that a fit with a non-truncated disc is preferred with $2.8 \sigma$ confidence. However, any alternative fit with a truncated disc would also require a smaller reflection fraction in order to accommodate the correspondingly narrower relativistic iron line component. The extra dilution resulting from this lower reflection fraction would increase the inferred intrinsic light crossing lag.

In this context, it is interesting to note that the largest lag measured here $(705 \pm 198 \mathrm{~s})$ is actually almost the same $(1037 \pm 455 \mathrm{~s}$; Zoghbi et al. 2013) as that measured in the higher mass MGC-5-23$16\left(8 \times 10^{7} \mathrm{M}_{\odot}\right.$; Ponti et al. 2012). From X-ray spectral analysis, the good agreement between the lags can be partially explained by the significantly lower reflection fraction found in MGC-5-23-16 ( $R=0.3$; Zoghbi et al. 2017). The dilution effect for MGC-5-23-16 will be higher than for NGC 5273, explaining why the observed lags in the two AGNs are comparable. Moreover, the different reflection fractions suggest that the two sources have different disc configurations. Given that the inferred truncation radius of MGC5-23-16 is of the order $\approx 50 R_{\mathrm{G}}$, these results seem to point towards a self-consistent picture.

The lag versus energy spectrum can also be used to extract physical information on the properties on the accretion flow. The energy dependence of the lag shows a gradual increase before a sharp drop at $8 \mathrm{keV}$. Such a trend is in good agreement with the expectation from disc reverberation (Cackett et al. 2014; Chainakun et al. 2019; Ingram et al. 2019). Moreover the energy at which the lag peaks does not correspond to the energy at which the iron line peaks in the energy spectrum ( $7 \mathrm{keV}$ against $6.4 \mathrm{keV})$. This was already observed for NGC 4151 by Zoghbi et al. (2012), and it is due to the different origin of the broad and narrow components in the time-averaged spectrum. While the first is explained in terms 
of an accretion disc very close to the central black hole (giving origin also to the measured lags), the latter is believed to originate from a more distant and cold material (Zoghbi et al. 2012; Zoghbi, Miller \& Cackett 2019).

The value at which the lag versus energy drops is known to be directly related to the inclination of the disc (Cackett et al. 2014). In particular, the more edge-on the source is, the higher the Dopplershifted contribution will be, and therefore the higher the energy of the drop. It is possible to show that if the peak of the lag is after $7 \mathrm{keV}$, the inclination is higher than $45^{\circ}$ (see fig. 13 in Cackett et al. 2014). The high inclination seems also to be in good agreement with the general orientation-dependent picture of Seyfert classification. According to that scenario Seyfert galaxies which show mainly narrow lines (e.g. Sy 1.5 or 1.8) have a higher inclination (and therefore obscuration) than standard Seyfert galaxies. Of course other parameters could also affect the appearance of broader lines, originating from closer regions to the central black hole (e.g. size of broad-line region or optical depth of the obscuring material). We note that a greater sample of spectral-timing measurements will permit in future to quantify the actual effect of the inferred accretion disc inclination on the optical lines.

\section{CONCLUSIONS}

We present a detailed characterization of the X-ray variability of the Seyfert 1.5 galaxy NGC 5273 from a 90 ks XMM-Newton observation. The source shows significant variability in the observations down to time-scales of 1000 s. Applying cross-spectral analysis, we found that the emission between 5 and $7 \mathrm{keV}$ lags the rest of the continuum by $\approx 1000 \mathrm{~s}$. Given also the presence of a significant emission line in the X-ray spectrum at $6.4 \mathrm{keV}$ we interpret this delay as the result of reverberation due to the illumination of the disc by X-rays from a hot central corona. From the shape of the lagenergy spectrum, we found that the inclination of the source should be $\gtrsim 45^{\circ}$, which is in good agreement with the classification of the source. The result presented here is a further confirmation of how powerful timing studies can be for probing the geometry of AGNs even in lower luminosity sources, which have not previously been explored with similar techniques. Given the long-term variability displayed by the source, new observations done at different flux levels could explore changes in geometry.

\section{ACKNOWLEDGEMENTS}

FV would like to thank Michiel van der Klis for the useful discussion and comments on the timing analysis of this data set. $\mathrm{IMcH}$ and FV thank STFC for support under grant ST/M001326/1. MP acknowledges Royal Society-SERB Newton International Fellowship support funded jointly by the Royal Society, UK and the Science and Engineering Board of India (SERB) through Newton-Bhabha Fund.

\section{REFERENCES}

Alston W. N., Done C., Vaughan S., 2014, MNRAS, 439, 1548

Bentz M. C. et al., 2014, ApJ, 796, 8

Caballero-García M. D., Papadakis I. E., Dovčiak M., Bursa M., Epitropakis A., Karas V., Svoboda J., 2018, MNRAS, 480, 2650

Cabanac C., Fender R. P., Dunn R. J. H., Körding E. G., 2009, MNRAS, 396, 1415

Cackett E. M., Zoghbi A., Reynolds C., Fabian A. C., Kara E., Uttley P., Wilkins D. R., 2014, MNRAS, 438, 2980
Cappi M. et al., 2006, A\&A, 446, 459

Chainakun P., Young A. J., 2017, MNRAS, 465, 3965

Chainakun P., Watcharangkool A., Young A. J., Hancock S., 2019, MNRAS, 487, 667

Cicone C. et al., 2014, A\&A, 562, A21

Dauser T., Garcia J., Wilms J., Böck M., Brenneman L. W., Falanga M., Fukumura K., Reynolds C. S., 2013, MNRAS, 430, 1694

Dauser T., Garcia J., Parker M. L., Fabian A. C., Wilms J., 2014, MNRAS, 444, L100

De Marco B., Ponti G., Cappi M., Dadina M., Uttley P., Cackett E. M., Fabian A. C., Miniutti G., 2013, MNRAS, 431, 2441

Emmanoulopoulos D., McHardy I. M., Papadakis I. E., 2011, MNRAS, 416, L94

Emmanoulopoulos D., Papadakis I. E., Dovčiak M., McHardy I. M., 2014, MNRAS, 439, 3931

Fabian A. C., 2012, ARA\&A, 50, 455

Fabian A. C., Rees M. J., Stella L., White N. E., 1989, MNRAS, 238 , 729

Fabian A. C. et al., 2009, Nature, 459, 540

García J., Dauser T., Reynolds C. S., Kallman T. R., McClintock J. E., Wilms J., Eikmann W., 2013, ApJ, 768, 146

García J. et al., 2014, ApJ, 782, 76

Graham A. W., Onken C. A., Athanassoula E., Combes F., 2011, MNRAS, 412,2211

Guilbert P. W., Rees M. J., 1988, MNRAS, 233, 475

Haardt F., Maraschi L., 1991, ApJ, 380, L51

Huppenkothen D. et al., 2019, ApJ, 881, 39

Ingram A., Mastroserio G., Dauser T., Hovenkamp P., van der Klis M., García J. A., 2019, MNRAS, 488, 324

Jenkins G. M., Watts D. G., 1969, Spectral Analysis and its Applications. Holden-Day, San Francisco

Kara E., Fabian A. C., Cackett E. M., Steiner J. F., Uttley P., Wilkins D. R., Zoghbi A., 2013a, MNRAS, 428, 2795

Kara E., Fabian A. C., Cackett E. M., Miniutti G., Uttley P., 2013b, MNRAS, 430, 1408

Kara E., Alston W. N., Fabian A. C., Cackett E. M., Uttley P., Reynolds C. S., Zoghbi A., 2016, MNRAS, 462, 511

Kawamuro T., Ueda Y., Tazaki F., Terashima Y., Mushotzky R., 2016, ApJ, 831,37

King A., Pounds K., 2015, ARA\&A, 53, 115

Lawrence A., Watson M. G., Pounds K. A., Elvis M., 1987, Nature, 325 , 694

Lobban A. P., Alston W. N., Vaughan S., 2014, MNRAS, 445, 3229

Markowitz A., Papadakis I., Arévalo P., Turner T. J., Miller L., Reeves J. N., 2007, ApJ, 656, 116

Martocchia A., Karas V., Matt G., 2000, MNRAS, 312, 817

McHardy I., Czerny B., 1987, Nature, 325, 696

McHardy I. M., Papadakis I. E., Uttley P., Page M. J., Mason K. O., 2004, MNRAS, 348, 783

McHardy I. M., Koerding E., Knigge C., Uttley P., Fender R. P., 2006 , Nature, 444, 730

McHardy I. M., Arévalo P., Uttley P., Papadakis I. E., Summons D. P., Brinkmann W., Page M. J., 2007, MNRAS, 382, 985

Osterbrock D. E., Martel A., 1993, ApJ, 414, 552

Padovani P. et al., 2017, A\&AR, 25, 2

Pahari M., McHardy I. M., Mallick L., Dewangan G. C., Misra R., 2017, MNRAS, 470, 3239

Polletta M., Bassani L., Malaguti G., Palumbo G. G. C., Caroli E., 1996, ApJS, 106, 399

Ponti G., Papadakis I., Bianchi S., Guainazzi M., Matt G., Uttley P., Bonilla N. F., 2012, A\&A, 542, A83

Ross R. R., Fabian A. C., 1993, MNRAS, 261, 74

Schawinski K., Thomas D., Sarzi M., Maraston C., Kaviraj S., Joo S.-J., Yi S. K., Silk J., 2007, MNRAS, 382, 1415

Tanaka Y. et al., 1995, Nature, 375, 659

Taylor C., Reynolds C. S., 2018, ApJ, 868, 109

Tonry J. L., Dressler A., Blakeslee J. P., Ajhar E. A., Fletcher A. B., Luppino G. A., Metzger M. R., Moore C. B., 2001, ApJ, 546, 681 
Trippe M. L., Crenshaw D. M., Deo R. P., Dietrich M., Kraemer S. B., Rafter S. E., Turner T. J., 2010, ApJ, 725, 1749

Uttley P., McHardy I. M., 2001, MNRAS, 323, L26

Uttley P., McHardy I. M., Papadakis I. E., 2002, MNRAS, 332, 231

Uttley P., McHardy I. M., Vaughan S., 2005, MNRAS, 359, 345

Uttley P., Cackett E. M., Fabian A. C., Kara E., Wilkins D. R., 2014, A\&AR, 22,72

Vaughan S., Edelson R., Warwick R. S., Uttley P., 2003, MNRAS, 345, 1271

Wilkins D. R., Cackett E. M., Fabian A. C., Reynolds C. S., 2016, MNRAS, 458,200
Zoghbi A., Uttley P., Fabian A. C., 2011, MNRAS, 412, 59

Zoghbi A., Fabian A. C., Reynolds C. S., Cackett E. M., 2012, MNRAS, 422, 129

Zoghbi A., Reynolds C., Cackett E. M., Miniutti G., Kara E., Fabian A. C., 2013, ApJ, 767, 121

Zoghbi A. et al., 2017, ApJ, 836, 2

Zoghbi A., Miller J. M., Cackett E., 2019, ApJ, 884, 26

This paper has been typeset from a $\mathrm{T}_{\mathrm{E}} \mathrm{X} / \mathrm{L} \mathrm{T} \mathrm{T}_{\mathrm{E}} \mathrm{X}$ file prepared by the author. 